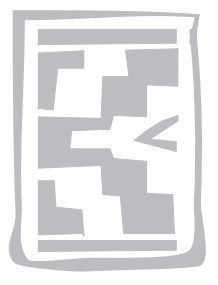

\title{
Small mammals as hosts of immature ixodid ticks
}

\author{
I.G. HORAK ${ }^{1,2}$, L.J. FOURIE ${ }^{2}$ and L.E.O. BRAACK ${ }^{3}$
}

\begin{abstract}
HORAK, I.G., FOURIE, L.J. \& BRAACK, L.E.O. 2005. Small mammals as hosts of immature ixodid ticks. Onderstepoort Journal of Veterinary Research, 72:255-261

Two hundred and twenty-five small mammals belonging to 16 species were examined for ticks in Free State, Mpumalanga and Limpopo Provinces, South Africa, and 18 ixodid tick species, of which two could only be identified to genus level, were recovered. Scrub hares, Lepus saxatilis, and Cape hares, Lepus capensis, harboured the largest number of tick species. In Free State Province Namaqua rock mice, Aethomys namaquensis, and four-striped grass mice, Rhabdomys pumilio, were good hosts of the immature stages of Haemaphysalis leachi and Rhipicephalus gertrudae, while in Mpumalanga and Limpopo Provinces red veld rats, Aethomys chrysophilus, Namaqua rock mice and Natal multimammate mice, Mastomys natalensis were good hosts of $H$. leachi and Rhipicephalus simus. Haemaphysalis leachi was the only tick recovered from animals in all three provinces.
\end{abstract}

Keywords: Immature ixodid ticks, Haemaphysalis leachi, Rhipicephalus gertrudae, Rhipicephalus simus, small mammals, South Africa

\section{INTRODUCTION}

A large number of surveys have focused on the role of small mammals as hosts of the immature stages of ixodid ticks in South Africa. The accent has been mainly on murid rodents (Rechav 1982; Howell, Petney \& Horak 1989; Horak, Fourie, Novellie \& Williams 1991; Fourie, Horak \& Van Den Heever 1992; Braack, Horak, Jordaan, Segerman \& Louw 1996; Horak \& Boomker 1998; Horak \& Cohen 2001; Petney, Horak, Howell \& Meyer 2004), but

1 Department of Veterinary Tropical Diseases, Faculty of Veterinary Science, University of Pretoria, Onderstepoort, 0110 South Africa. E-mail: ivan.horak@up.ac.za

2 Department of Zoology and Entomology, University of the Free State, Bloemfontein, 9301 South Africa

3 Scientific Services, South African National Parks, Kruger National Park, Skukuza, 1350 South Africa. Present address: P.O. Box 2550, Brooklyn Square, 0075 South Africa

Accepted for publication 14 June 2005-Editor elephant shrews (Stampa 1959; Fourie et al. 1992; Fourie, Horak, Kok \& Van Zyl 2002), hares and rabbits (Stampa 1959; Horak, Sheppey, Knight \& Beuthin 1986; Horak \& Fourie 1991; Horak et al. 1991; Horak, Spickett, Braack \& Penzhorn 1993; Horak, Spickett, Braack, Penzhorn, Bagnall \& Uys 1995; Maclvor \& Horak 2003), rock dassies (Horak \& Fourie 1986; Horak et al. 1991), and small carnivores (Horak, Chaparro, Beaucournu \& Louw 1999; Horak, Braack, Fourie \& Walker 2000) have also been examined.

The adults of some of the tick species that infest these small animals as larvae or nymphs are important vectors of disease or toxins to domestic livestock, whereas others are of little or no economic consequence. Thus murid rodents are among the preferred hosts of the immature stages of Haemaphysalis leachi, whose adults parasitize and transmit canine babesiosis to domestic dogs (Lewis, Penzhorn, Lopez-Rebollar \& De Waal 1996), and of 
Rhipicephalus simus, whose adults parasitize cattle, horses and domestic dogs and transmit anaplasmosis to cattle and produce a toxin causing paralysis in calves and lambs (Walker, Keirans \& Horak 2000). Rock elephant shrews, Elephantulus myurus, are the hosts most favoured by the immature stages of Ixodes rubicundus and Rhipicephalus warburtoni, whose adults cause paralysis in sheep and goats (Stampa 1959; Walker et al. 2000), and scrub hares, Lepus saxatilis, by the immature stages of Hyalomma marginatum rufipes and Hyalomma truncatum, whose adults are parasites of domestic cattle, sheep and goats. The former tick transmits anaplasmosis and Babesia occultans to cattle, and the latter secretes a toxin that is the cause of sweating sickness in these animals (Walker, Bouattour, Camicas, Estrada-Peña, Horak, Latif, Pegram \& Preston 2003). The objective of the present paper is to present recent data on the ixodid ticks that infest small mammals in three of the nine provinces of South Africa, and to supply the geographic coordinates of the localities at which the ticks were collected for future mapping purposes.

\section{MATERIALS AND METHODS}

Two hundred and twenty-five small mammals, belonging to 16 species, were collected in Free State, Mpumalanga and Limpopo Provinces, South Africa and processed for tick recovery as described by Horak et al. (1986). One hundred and sixty-three of these animals belonging to 10 species were examined in Free State Province and 62 belonging to 11 species were examined in Mpumalanga and Limpopo Provinces (Table 1).

\section{RESULTS AND DISCUSSION}

The ticks collected in Free State Province are summarized in Tables 2 and 3 , and those from animals in the north-eastern regions of Mpumalanga and Limpopo Provinces in Tables 4 and 5.

Twelve tick species, of which two could be identified only to genus level, were recovered in Free State Province and nine in Mpumalanga and Limpopo Provinces. Only $H$. leachi was collected in all three provinces, while $H$. truncatum and Rhipicephalus evertsi evertsi were collected in two, namely Free State and Mpumalanga Provinces.

Scrub hares, L. saxatilis, and Cape hares, Lepus capensis, were infested with the largest number of tick species. In Free State Province Namaqua rock mice, Aethomys namaquensis, and four-striped grass mice, Rhabdomys pumilio, were good hosts of the immature stages of $H$. leachi and Rhipicephalus gertrudae, while in Mpumalanga and Limpopo Provinces red veld rats, Aethomys chrysophilus, Namaqua rock mice and Natal multimammate mice, Mastomys natalensis, were good hosts of $H$. leachi and $R$. simus, a tick anagolous to $R$. gertrudae.

\section{Dermacentor rhinocerinus}

The hosts of the immature stages of this tick were unknown until Horak \& Cohen (2001) collected larvae and nymphs from rodents in Mpumalanga Province. For the sake of completeness we have repeated their findings here because other tick species, recovered from the same hosts, were not listed in the earlier publication. Dermacentor rhinocerinus is a host-specific parasite of rhinoceroses (Keirans 1993), of which there are several in the Mthethomusha Reserve, Mpumalanga Province. These animals must have been the source of infestation reflected in the burdens of immature ticks of five of the 38 rodents examined in the reserve (Table 4). There are rhinoceroses in the Willem Pretorius Nature Reserve in Free State Province, but it is not known whether $D$. rhinocerinus is also present.

\section{Haemaphysalis leachi}

Domestic dogs and the larger wild felids are the preferred hosts of adult $H$. leachi, while its immature stages occur mainly on murid rodents (Norval 1984; Braack et al. 1996; Horak et al. 2000). Its widespread distribution in the present survey confirms Norval's (1984) assertion that provided hosts for its adults and immature stages are present, almost any locality in southern Africa can be regarded as suitable. Furthermore the large range of carnivore and rodent species parasitized by its adult and immature stages, respectively (Norval 1984; Horak et al. 2000; Tables 2 and 4) and their widespread distribution, ensure that should $H$. leachi be introduced into a region it would readily become established. This assumption is corroborated by its extensive but discontinuous distribution in South Africa (Howell, Walker \& Nevill 1978).

Haemaphysalis leachi transmits Babesia canis, the cause of canine babesiosis in domestic dogs in South Africa (Lewis et al. 1996). Judging by the host preferences of the adult and immature stages of the tick it would seem that transmission mostly takes place via the adults with infection passing transovarially from adult ticks of the previous generation without it being lost when the immature stages feed on rodents. 
TABLE 1 Small mammals and the localities at which they were examined for ixodid ticks

\begin{tabular}{|c|c|c|c|}
\hline \multicolumn{3}{|l|}{ Small mammal species } & \multirow[b]{2}{*}{ Province, locality and coordinates } \\
\hline Common name & Scientific name & $\begin{array}{l}\text { No. } \\
\text { examined }\end{array}$ & \\
\hline Tree squirrel & Paraxerus cepapi & 2 & 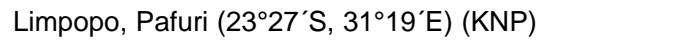 \\
\hline $\begin{array}{l}\text { Short-tailed pouched } \\
\text { mouse }\end{array}$ & $\begin{array}{l}\text { Saccostomys } \\
\text { campestris }\end{array}$ & $\begin{array}{l}1 \\
3\end{array}$ & $\begin{array}{l}\text { Free State, Tussen-die-Riviere NR }\left(30^{\circ} 29^{\prime} \mathrm{S}, 26^{\circ} 15^{\prime} \mathrm{E}\right) \\
\text { Limpopo, Pafuri }\left(23^{\circ} 27^{\prime} \mathrm{S}, 31^{\circ} 19^{\prime} \mathrm{E}\right)(\mathrm{KNP})\end{array}$ \\
\hline Bushveld gerbil & Tatera leucogaster & $\begin{array}{l}5 \\
4 \\
1\end{array}$ & $\begin{array}{l}\text { Free State, Sandveld NR }\left(27^{\circ} 38^{\prime} \mathrm{S} 25^{\circ} 42^{\prime} \mathrm{E}\right) \\
\text { Mpumalanga, Mtethomusha NR }\left(25^{\circ} 29^{\prime} \mathrm{S}, 31^{\circ} 17^{\prime} \mathrm{E}\right) \\
\text { Mpumalanga, Pretoriuskop }\left(25^{\circ} 10^{\prime} \mathrm{S}, 31^{\circ} 16^{\prime} \mathrm{E}\right) \text { (KNP) }\end{array}$ \\
\hline Red veld rat & $\begin{array}{l}\text { Aethomys } \\
\text { chrysophilus }\end{array}$ & $\begin{array}{r}10 \\
1 \\
8\end{array}$ & $\begin{array}{l}\text { Mpumalanga, Mthethomusha NR }\left(25^{\circ} 29^{\prime} \mathrm{S}, 31^{\circ} 17^{\prime} \mathrm{E}\right) \\
\text { Mpumalanga, Berg-en-Dal }\left(25^{\circ} 25^{\prime} \mathrm{S}, 31^{\circ} 27^{\prime} \mathrm{E}\right)(\mathrm{KNP}) \\
\text { Limpopo, Pafuri }\left(23^{\circ} 27^{\prime} \mathrm{S}, 31^{\circ} 19^{\prime} \mathrm{E}\right)(\mathrm{KNP})\end{array}$ \\
\hline Namaqua rock mouse & $\begin{array}{l}\text { Aethomys } \\
\text { namaquensis }\end{array}$ & $\begin{array}{r}17 \\
21 \\
34 \\
5 \\
5 \\
15 \\
6 \\
1 \\
3\end{array}$ & $\begin{array}{l}\text { Free State, "Preezfontein" }\left(29^{\circ} 50^{\prime} \mathrm{S}, 2^{\circ} 23^{\prime} \mathrm{E}\right) \\
\text { Free State, Tussen-die-Riviere NR }\left(30^{\circ} 29^{\prime} \mathrm{S}, 26^{\circ} 15^{\prime} \mathrm{E}\right) \\
\text { Free State, Welbedacht Dam }\left(29^{\circ} 51^{\prime} \mathrm{S}, 26^{\circ} 53^{\prime} \mathrm{E}\right) \\
\text { Free State, Zandbult (locality unknown) } \\
\text { Free State, Wolhuterskop }\left(28^{\circ} 14^{\prime} \mathrm{S}, 28^{\circ} 18^{\prime} \mathrm{E}\right) \\
\text { Free State, Willem Pretorius NR }\left(28^{\circ} 19^{\prime} \mathrm{S}, 27^{\circ} 15^{\prime} \mathrm{E}\right) \\
\text { Mpumalanga, Mthethomusha NR }\left(25^{\circ} 29^{\prime} \mathrm{S}, 31^{\circ} 17^{\prime} \mathrm{E}\right) \\
\text { Limpopo, Tshalungwa }\left(22^{\circ} 33^{\prime} \mathrm{S}, 31^{\circ} 05^{\prime} \mathrm{E}\right) \text { (KNP) } \\
\text { Limpopo, Pafuri }\left(23^{\circ} 27^{\prime} \mathrm{S}, 31^{\circ} 19^{\prime} \mathrm{E}\right)(\mathrm{KNP})\end{array}$ \\
\hline Single-striped mouse & Lemniscomys rosalia & 1 & Mpumalanga, Mthethomusha NR $\left(25^{\circ} 29^{\prime} \mathrm{S}, 31^{\circ} 17^{\prime} \mathrm{E}\right)$ \\
\hline Multimammate mouse & Mastomys coucha & 10 & Mpumalanga, Mthethomusha NR $\left(25^{\circ} 29^{\prime} \mathrm{S}, 31^{\circ} 17^{\prime} \mathrm{E}\right)$ \\
\hline $\begin{array}{l}\text { Natal multimammate } \\
\text { mouse }\end{array}$ & Mastomys natalensis & $\begin{array}{l}6 \\
1 \\
1 \\
1\end{array}$ & $\begin{array}{l}\text { Mpumalanga, Mthethomusha NR }\left(25^{\circ} 29^{\prime} \mathrm{S}, 31^{\circ} 17^{\prime} \mathrm{E}\right) \\
\text { Limpopo, Dube Station (KNP) } \\
\text { Limpopo, Mashikiri }\left(22^{\circ} 35^{\prime} \mathrm{S}, 31^{\circ} 11^{\prime} \mathrm{E}\right)(\mathrm{KNP}) \\
\text { Limpopo, Pafuri KNP }\left(23^{\circ} 27^{\prime} \mathrm{S}, 31^{\circ} 19^{\prime} \mathrm{E}\right)\end{array}$ \\
\hline Pigmy mouse & Mus minutoides & 2 & Free State, Welbedacht Dam (29॰51'S, $\left.26^{\circ} 53^{\prime} E\right)$ \\
\hline Black rat & Rattus rattus & $\begin{array}{l}1 \\
1\end{array}$ & $\begin{array}{l}\text { Free State, Sandveld NR }\left(27^{\circ} 38^{\prime} \mathrm{S} 25^{\circ} 42^{\prime} \mathrm{E}\right) \\
\text { Mpumalanga, Skukuza }\left(24^{\circ} 58^{\prime} \mathrm{S}, 31^{\circ} 36^{\prime} \mathrm{E}\right)(\mathrm{KNP})\end{array}$ \\
\hline $\begin{array}{l}\text { Four-striped grass } \\
\text { mouse }\end{array}$ & Rhabdomys pumilio & $\begin{array}{l}7 \\
5 \\
4 \\
7 \\
9 \\
4\end{array}$ & $\begin{array}{l}\text { Free State, Tussen-die-Riviere NR }\left(30^{\circ} 29^{\prime} \mathrm{S}, 26^{\circ} 15^{\prime} \mathrm{E}\right) \\
\text { Free State, Platberg }\left(28^{\circ} 16^{\prime} \mathrm{S}, 29^{\circ} 10^{\prime} \mathrm{E}\right) \\
\text { Free State, Welbedacht Dam }\left(29^{\circ} 51^{\prime} \mathrm{S}, 26^{\circ} 53^{\prime} \mathrm{E}\right) \\
\text { Free State, Zandbult (locality unknown) } \\
\text { Free State, Wolhuterskop }\left(28^{\circ} 14^{\prime} \mathrm{S}, 28^{\circ} 18^{\prime} \mathrm{E}\right) \\
\text { Free State, Golden Gate NP }\left(28^{\circ} 31^{\prime} \mathrm{S}, 28^{\circ} 37^{\prime} \mathrm{E}\right)\end{array}$ \\
\hline Angoni swamp rat & Otomys angoniensis & 1 & Mpumalanga, Mthethomusha NR $\left(25^{\circ} 29^{\prime} \mathrm{S}, 31^{\circ} 17^{\prime} \mathrm{E}\right)$ \\
\hline Swamp rat & Otomys irroratus & 1 & Free State, Golden Gate NP ( $\left.28^{\circ} 31^{\prime} \mathrm{S}, 2^{\circ} 37^{\prime} \mathrm{E}\right)$ \\
\hline Spring hare & Pedetes capensis & 14 & Free State, Sandveld NR (29॰51'S, $\left.26^{\circ} 53^{\prime} E\right)$ \\
\hline Cape hare & Lepus capensis & $\begin{array}{l}1 \\
2\end{array}$ & $\begin{array}{l}\text { Free State, Tussen-die-Riviere NR }\left(30^{\circ} 29^{\prime} \mathrm{S}, 26^{\circ} 15^{\prime} \mathrm{E}\right) \\
\text { Free State, Willem Pretorius NR }\left(28^{\circ} 19^{\prime} \mathrm{S}, 27^{\circ} 15^{\prime} \mathrm{E}\right)\end{array}$ \\
\hline Scrub hare & Lepus saxatilis & $\begin{array}{l}1 \\
2 \\
1\end{array}$ & $\begin{array}{l}\text { Free State, Tussen-die-Riviere NR }\left(30^{\circ} 29^{\prime} \mathrm{S}, 26^{\circ} 15^{\prime} \mathrm{E}\right) \\
\text { Free State, Willem Pretorius NR }\left(28^{\circ} 19^{\prime} \mathrm{S}, 27^{\circ} 15^{\prime} \mathrm{E}\right) \\
\text { Mpumalanga, Mthethomusha NR }\left(25^{\circ} 29^{\prime} \mathrm{S}, 31^{\circ} 17^{\prime} \mathrm{E}\right)\end{array}$ \\
\hline
\end{tabular}

$\mathrm{KNP}=$ Kruger National Park

$\mathrm{NP}=$ National Park

$\mathrm{NR}=$ Nature Reserve 
Small mammals as hosts of immature ixodid ticks

TABLE 2 Ticks, other than Rhipicephalus species, on small mammals in Free State Province

\begin{tabular}{|c|c|c|c|c|}
\hline \multirow{2}{*}{ Tick and host species } & \multirow{2}{*}{$\begin{array}{l}\text { No. examined } \\
\text { (No. infested) }\end{array}$} & \multicolumn{3}{|c|}{ Number of ticks recovered } \\
\hline & & Larvae & Nymphs & Total \\
\hline $\begin{array}{l}\text { Amblyomma marmoreum } \\
\text { Lepus saxatilis }\end{array}$ & $3(1)$ & 4 & 17 & 21 \\
\hline $\begin{array}{l}\text { Haemaphysalis leachi } \\
\text { Aethomys namaquensis } \\
\text { Rhabdomys pumilio }\end{array}$ & $\begin{array}{l}97(38) \\
36(9)\end{array}$ & $\begin{array}{l}63 \\
72\end{array}$ & $\begin{array}{r}35 \\
5\end{array}$ & $\begin{array}{l}98 \\
77\end{array}$ \\
\hline $\begin{array}{l}\text { Hyalomma marginatum } \\
\text { Rhabdomys pumilio } \\
\text { Lepus capensis } \\
\text { Lepus saxatilis }\end{array}$ & $\begin{array}{r}36(1) \\
3(1) \\
3(2)\end{array}$ & $\begin{array}{r}1 \\
12 \\
4\end{array}$ & $\begin{array}{l}0 \\
6 \\
9\end{array}$ & $\begin{array}{r}1 \\
18 \\
13\end{array}$ \\
\hline $\begin{array}{l}\text { Hyalomma truncatum } \\
\text { Aethomys namaquensis } \\
\text { Rhabdomys pumilio } \\
\text { Tatera leucogaster } \\
\text { Lepus capensis } \\
\text { Lepus saxatilis }\end{array}$ & $\begin{array}{r}97(2) \\
36(1) \\
5(1) \\
3(2) \\
3(2)\end{array}$ & $\begin{array}{r}2 \\
1 \\
4 \\
4 \\
33\end{array}$ & $\begin{array}{r}0 \\
0 \\
0 \\
7 \\
39\end{array}$ & $\begin{array}{r}2 \\
1 \\
4 \\
11 \\
72\end{array}$ \\
\hline $\begin{array}{l}\text { Ixodes rubicundus } \\
\text { Aethomys namaquensis } \\
\text { Lepus saxatilis }\end{array}$ & $\begin{array}{r}97(6) \\
3(1)\end{array}$ & $\begin{array}{r}16 \\
0\end{array}$ & $\begin{array}{l}2 \\
1\end{array}$ & $\begin{array}{r}18 \\
1\end{array}$ \\
\hline $\begin{array}{l}\text { Ixodes sp. } \\
\text { Aethomys namaquensis } \\
\text { Rhabdomys pumilio }\end{array}$ & $\begin{array}{l}97(8) \\
36(2)\end{array}$ & $\begin{array}{l}2 \\
1\end{array}$ & $\begin{array}{l}7 \\
1\end{array}$ & $\begin{array}{l}9 \\
2\end{array}$ \\
\hline $\begin{array}{l}\text { Margaropus winthemi } \\
\text { Rhabdomys pumilio } \\
\text { Lepus saxatilis }\end{array}$ & $\begin{array}{r}36(1) \\
3(1)\end{array}$ & $\begin{array}{l}1 \\
4\end{array}$ & $\begin{array}{l}0 \\
1\end{array}$ & $\begin{array}{l}1 \\
5\end{array}$ \\
\hline
\end{tabular}

TABLE 3 Rhipicephalus species on small mammals in Free State Province

\begin{tabular}{|c|c|c|c|c|}
\hline \multirow{2}{*}{ Tick and host species } & \multirow{2}{*}{$\begin{array}{l}\text { No. examined } \\
\text { (No. infested) }\end{array}$} & \multicolumn{3}{|c|}{ Number of ticks recovered } \\
\hline & & Larvae & Nymphs & Total \\
\hline $\begin{array}{l}\text { Rhipicephalus evertsi evertsi } \\
\text { Aethomys namaquensis } \\
\text { Tatera leucogaster } \\
\text { Lepus capensis } \\
\text { Lepus saxatilis }\end{array}$ & $\begin{array}{r}97(2) \\
5(1) \\
3(2) \\
3(2)\end{array}$ & $\begin{array}{r}2 \\
1 \\
6 \\
31\end{array}$ & $\begin{array}{r}0 \\
0 \\
17 \\
61\end{array}$ & $\begin{array}{r}2 \\
1 \\
23 \\
92\end{array}$ \\
\hline $\begin{array}{l}\text { Rhipicephalus gertrudae } \\
\text { Aethomys namaquensis } \\
\text { Rhabdomys pumilio }\end{array}$ & $\begin{array}{l}97(29) \\
36(11)\end{array}$ & $\begin{array}{l}99 \\
50\end{array}$ & $\begin{array}{r}48 \\
6\end{array}$ & $\begin{array}{r}147 \\
56\end{array}$ \\
\hline $\begin{array}{l}\text { Rhipicephalus lounsburyi } \\
\text { Rhabdomys pumilio }\end{array}$ & $36(1)$ & 1 & 1 & 2 \\
\hline $\begin{array}{l}\text { Rhipicephalus warburtoni } \\
\text { Aethomys namaquensis } \\
\text { Pedetes capensis } \\
\text { Lepus capensis } \\
\text { Lepus saxatilis }\end{array}$ & $\begin{aligned} 97 & (11) \\
14 & (14) \\
3 & (1) \\
3 & (3)\end{aligned}$ & $\begin{array}{r}39 \\
0 \\
6 \\
22\end{array}$ & $\begin{array}{r}0 \\
34 \\
0 \\
1\end{array}$ & $\begin{array}{l}39 \\
34 \\
6+3 \sigma^{x} \sigma^{x} \\
23+7 \sigma^{x} \sigma^{x}\end{array}$ \\
\hline $\begin{array}{l}\text { Rhipicephalus sp. } \\
\text { Aethomys namaquensis } \\
\text { Rhabdomys pumilio } \\
\text { Tatera leucogaster }\end{array}$ & $\begin{array}{r}97(2) \\
36(1) \\
5(3)\end{array}$ & $\begin{array}{l}1 \\
1 \\
2\end{array}$ & $\begin{array}{r}1 \\
0 \\
12\end{array}$ & $\begin{array}{r}2 \\
1 \\
14\end{array}$ \\
\hline
\end{tabular}


I.G. HORAK, L.J. FOURIE \& L.E.O. BRAACK

TABLE 4 Ticks, other than Rhipicephalus species, on small mammals in north-eastern Mpumalanga and Limpopo Provinces

\begin{tabular}{|c|c|c|c|c|}
\hline \multirow{2}{*}{ Tick and host species } & \multirow{2}{*}{$\begin{array}{l}\text { No. examined } \\
\text { (No. infested) }\end{array}$} & \multicolumn{3}{|c|}{ Number of ticks recovered } \\
\hline & & Larvae & Nymphs & Total \\
\hline $\begin{array}{l}\text { Amblyomma hebraeum } \\
\text { Mastomys natalensis }\end{array}$ & $9(1)$ & 1 & 0 & 1 \\
\hline $\begin{array}{l}\text { Dermacentor rhinocerinus } \\
\text { Aethomys chrysophilus } \\
\text { Mastomys natalensis } \\
\text { Tatera leucogaster }\end{array}$ & $\begin{array}{r}19(1) \\
9(1) \\
5(3)\end{array}$ & $\begin{array}{l}1 \\
0 \\
3\end{array}$ & $\begin{array}{l}0 \\
1 \\
2\end{array}$ & $\begin{array}{l}1 \\
1 \\
5\end{array}$ \\
\hline $\begin{array}{l}\text { Haemaphysalis leachi } \\
\text { Aethomys chrysophilus } \\
\text { Aethomys namaquensis } \\
\text { Mastomys natalensis } \\
\text { Mastomys coucha } \\
\text { Saccostomys campestris } \\
\text { Paraxerus cepapi }\end{array}$ & $\begin{array}{r}19(11) \\
10(6) \\
9(2) \\
10(2) \\
3(3) \\
2(1)\end{array}$ & $\begin{array}{r}17 \\
17 \\
0 \\
3 \\
1 \\
2\end{array}$ & $\begin{array}{r}29 \\
7 \\
8 \\
1 \\
4 \\
0\end{array}$ & $\begin{array}{r}46 \\
24 \\
8 \\
4 \\
5 \\
2\end{array}$ \\
\hline $\begin{array}{l}\text { Hyalomma truncatum } \\
\text { Lepus saxatilis }\end{array}$ & $1(1)$ & 0 & 14 & 14 \\
\hline $\begin{array}{l}\text { Rhipicephalus (Boophilus) decoloratus } \\
\text { Aethomys chrysophilus }\end{array}$ & $19(1)$ & 1 & 0 & 1 \\
\hline
\end{tabular}

TABLE 5 Rhipicephalus species on small mammals in north-eastern Mpumalanga and Limpopo Provinces

\begin{tabular}{|c|c|c|c|c|}
\hline \multirow{2}{*}{ Tick and host species } & \multirow{2}{*}{$\begin{array}{l}\text { No. examined } \\
\text { (No. infested) }\end{array}$} & \multicolumn{3}{|c|}{ Number of ticks recovered } \\
\hline & & Larvae & Nymphs & Total \\
\hline $\begin{array}{l}\text { Rhipicephalus appendiculatus } \\
\text { Mastomys natalensis } \\
\text { Lepus saxatilis }\end{array}$ & $\begin{array}{l}9(1) \\
1(1)\end{array}$ & $\begin{array}{l}1 \\
0\end{array}$ & $\begin{array}{l}0 \\
6\end{array}$ & $\begin{array}{l}1 \\
6\end{array}$ \\
\hline $\begin{array}{l}\text { Rhipicephalus evertsi evertsi } \\
\text { Lepus saxatilis }\end{array}$ & $1(1)$ & 0 & 5 & 5 \\
\hline $\begin{array}{l}\text { Rhipicephalus simus } \\
\text { Aethomys chrysophilus } \\
\text { Aethomys namaquensis } \\
\text { Mastomys natalensis } \\
\text { Mastomys coucha } \\
\text { Otomys angoniensis } \\
\text { Lemniscomys rosalia } \\
\text { Saccostomys campestris } \\
\text { Rattus rattus } \\
\text { Tatera leucogaster } \\
\text { Paraxerus cepapi }\end{array}$ & $\begin{aligned} 19 & (15) \\
10 & (10) \\
9 & (5) \\
10 & (1) \\
1 & (1) \\
1 & (1) \\
3 & (1) \\
1 & (1) \\
5 & (2) \\
2 & (2)\end{aligned}$ & $\begin{array}{r}106 \\
112 \\
101 \\
1 \\
11 \\
9 \\
1 \\
1 \\
2 \\
11\end{array}$ & $\begin{array}{r}48 \\
15 \\
9 \\
0 \\
10 \\
3 \\
0 \\
0 \\
1 \\
1\end{array}$ & $\begin{array}{r}154 \\
127 \\
110 \\
1 \\
21 \\
12 \\
1 \\
1 \\
3 \\
12\end{array}$ \\
\hline $\begin{array}{l}\text { Rhipicephalus zambeziensis } \\
\text { Paraxerus cepapi }\end{array}$ & $2(2)$ & 11 & 18 & 29 \\
\hline
\end{tabular}

\section{Hyalomma species}

The immature stages of $H$. marginatum rufipes and $H$. marginatum turanicum infest Cape hares, scrub hares and ground frequenting birds, and those of $H$. truncatum hares, gerbils and murid rodents (Rechav,
Zeederberg \& Zeller 1987; Horak \& Fourie 1991; Horak et al. 1991; Braack et al. 1996). The distributions of $H$. marginatum rufipes and $H$. marginatum turanicum overlap in the southern Free State (Howell et al. 1978), and as we are unable to differentiate between their immature stages we have assigned 
only the specific epithet marginatum to the ticks collected from hares and a four-striped grass mouse examined in this region. Both hare species were infested with the larvae and nymphs of $H$. truncatum and three rodent species with only the larvae of this tick. The immature stages of the three Hyalomma ticks are present on their preferred hosts from early autumn to early summer (Horak et al. 1991; 1993; Horak \& Fourie 1991), and their presence in any study would thus be influenced by the season in which host animals are examined.

\section{Rhipicephalus evertsi evertsi}

The small number of rodents infested, and then only with larvae of this two-host tick, indicates that in contrast to hares, on which both larvae and nymphs were present, they are not good hosts. Although a large variety of domestic and wild ruminants are infested with $R$. evertsi evertsi, the preferred hosts of all stages of development are domestic and wild equids (Walker et al. 2000).

\section{Rhipicephalus gertrudae}

This tick replaces $R$. simus in the winter rainfall regions of south-western Western Cape Province and in the more arid regions of this province and Northern Cape Province as well as in the central and western regions of Free State Province (Walker et al. 2000). Its adult and immature stages have much the same host preference as $R$. simus, but in addition, the adults seem to favour primates, including humans (Brain \& Bohrmann 1992; Walker et al. 2000; Horak, Fourie, Heyne, Walker \& Needham 2002). The recovery of its larvae and nymphs only from $A$. namaquensis and $R$. pumilio and not from other small mammals within its distribution range does not necessarily reflect a host preference for these species, but may be influenced by the localities and seasons in which the mice were examined.

\section{Rhipicephalus lounsburyi}

The higher mountainous regions of the Eastern Cape Province are one of the preferred habitats of this tick (Walker et al. 2000). It has not previously been recorded in Free State Province, but Platberg, the locality at which it was collected from a fourstriped grass mouse, forms part of the same mountain range in which it has been collected in the Eastern Cape Province. The adults attach around the feet of their antelope and sheep hosts, while the only known host of its immature stages is a fourstriped grass mouse, from which a single nymph was collected (Walker et al. 2000).

\section{Rhipicephalus simus}

Of all species collected in the two northern provinces, the immature stages of $R$. simus has the largest host range, and judging by the numbers of larvae and the fact that nymphs also were recovered, most of the small mammal species examined could be considered as suitable hosts. Although cattle are frequently infested, the adults are parasites of the larger carnivore species, including domestic dogs, and of large monogastric animals such as zebras, rhinoceroses and warthogs (Horak et al. 2000; Walker et al. 2000).

\section{Rhipicephalus warburtoni}

The distribution of $R$. warburtoni is virtually confined to Free State Province (Walker et al. 2000). All stages of development prefer hares as hosts, while the adults are found on domestic and wild ruminants and the immature stages on rock elephant shrews (Walker et al. 2000). Its presence on all the spring hares, Pedetes capensis, and on some of the Namaqua rock mice examined indicates that these animals may also be suitable hosts. The adults, that at the time were identified as belonging to a species similar to Rhipicephalus pravus, produce a toxin causing paralysis in goat kids in the spring and early summer (Fourie, Horak \& Marais 1988).

\section{Other species}

With the possible exception of those on scrub hares, we consider the remaining species to be accidental parasites or "stragglers" on the small mammals. The immature stages of Amblyomma hebraeum and Amblyomma marmoreum parasitize scrub hares and small carnivores (Horak et al. 1995; Horak et al. 2000), but are hardly ever found on rodents (Howell et al. 1989; Horak et al. 1991; Braack et al. 1996; Petney et al. 2004). The immature stages of $I$. rubicundus prefer rock elephant shrews and Smith's red rock rabbits, Pronolagus rupestris (Stampa 1959; Fourie et al. 1992), and the one-host ticks, Margaropus winthemi and Rhipicephalus (Boophilus) decoloratus, are parasites of large herbivores (Howell et al. 1978).

\section{ACKNOWLEDGEMENTS}

We are most grateful to the farmers, landowners and nature reserve managers for permission to collect small mammals on the properties under their jurisdiction. The assistance of Mr E.J. Williams, Ms M. Cohen and Mr J. Sithole with the collection of ticks from many of the animals is greatly appreciated. 


\section{REFERENCES}

BRAACK, L.E.O., HORAK, I.G., JORDAAN, LEONORA C., SEGERMAN, JOYCE \& LOUW, J.P. 1996. The comparative host status of red veld rats (Aethomys chrysophilus) and bushveld gerbils (Tatera leucogaster) for epifaunal arthropods in the southern Kruger National Park, South Africa. Onderstepoort Journal of Veterinary Research, 63:149-158.

BRAIN, C. \& BOHRMANN, R. 1992. Tick infestation of baboons (Papio ursinus) in the Namib Desert. Journal of Wildlife Diseases, 28:188-191.

FOURIE, L.J., HORAK, I.G. \& MARAIS, L. 1988. An undescribed Rhipicephalus species associated with field paralysis of Angora goats. Journal of the South African Veterinary Association, 59:47-49.

FOURIE, L.J., HORAK, I.G. \& VAN DEN HEEVER, J.J. 1992. The relative host status of rock elephant shrews Elephantulus myurus and Namaqua rock mice Aethomys namaquensis for economically important ticks. South African Journal of Zoology, 27:108-114.

FOURIE, L.J., HORAK, I.G., KOK, D.J. \& VAN ZYL, W. 2002. Hosts, seasonal occurrence and life cycle of Rhipicentor nuttalli (Acari: Ixodidae). Onderstepoort Journal of Veterinary Research, 69:177-187.

HORAK, I.G. \& FOURIE, L.J. 1986. Parasites of domestic and wild animals in South Africa. XIX. Ixodid ticks and fleas on rock dassies (Procavia capensis) in the Mountain Zebra National Park. Onderstepoort Journal of Veterinary Research, 53:123-126.

HORAK, I.G., SHEPPEY, K., KNIGHT, M.M. \& BEUTHIN, C.L. 1986. Parasites of domestic and wild animals in South Africa. XXI. Arthropod parasites of vaal ribbok, bontebok and scrub hares in the western Cape Province. Onderstepoort Journal of Veterinary Research, 53:187-197.

HORAK, I.G., FOURIE, L.J., NOVELLIE, P.A. \& WILLIAMS, E.J. 1991. Parasites of domestic and wild animals in South Africa. XXVI. The mosaic of ixodid tick infestations on birds and mammals in the Mountain Zebra National Park. Onderstepoort Journal of Veterinary Research, 58:125-136.

HORAK, I.G. \& FOURIE, L.J. 1991. Parasites of domestic and wild animals in South Africa. XXIX. Ixodid ticks on hares in the Cape Province and on hares and red rock rabbits in the Orange Free State. Onderstepoort Journal of Veterinary Research, 58:261-270.

HORAK, I.G., SPICKETT, A.M., BRAACK, L.E.O. \& PENZHORN, B.L. 1993. Parasites of domestic and wild animals in South Africa. XXXII. Ixodid ticks on scrub hares in the Transvaal. Onderstepoort Journal of Veterinary Research, 60:163-174.

HORAK, I.G., SPICKETT, A.M., BRAACK, L.E.O., PENZHORN, B.L., BAGNALL, R.J. \& UYS, A.C. 1995. Parasites of domestic and wild animals in South Africa. XXXIII. Ixodid ticks on scrub hares in the north-eastern regions of Northern and Eastern Transvaal and of KwaZulu-Natal. Onderstepoort Journal of Veterinary Research, 62:123-131.

HORAK, I.G. \& BOOMKER, J. 1998. Parasites of domestic and wild animals in South Africa. XXXV. Ixodid ticks and bot fly larvae in the Bontebok National Park. Onderstepoort Journal of Veterinary Research, 65:205-211.

HORAK, I.G., CHAPARRO, F., BEAUCOURNU, J.-C. \& LOUW, J.P. 1999. Parasites of domestic and wild animals in South Africa. XXXVI. Arthropod parasites of yellow mongooses, Cynictis penicillata (G. Cuvier, 1829). Onderstepoort Journal of Veterinary Research, 66:33-38.
HORAK, I.G., BRAACK, L.E.O., FOURIE, L.J. \& WALKER, JANE B. 2000. Parasites of domestic and wild animals in South Africa. XXXVIII. Ixodid ticks collected from 23 wild carnivore species. Onderstepoort Journal of Veterinary Research, 67: 239-250.

HORAK, I.G. \& COHEN, MARLENE. 2001. Hosts of the immature stages of the rhinoceros tick, Dermacentor rhinocerinus (Acari: Ixodidae). Onderstepoort Journal of Veterinary Research, 68:75-77.

HORAK, I.G., FOURIE, L.J., HEYNE, HELOISE, WALKER, JANE B. \& NEEDHAM, G.R. 2002. Ixodid ticks feeding on humans in South Africa: with notes on preferred hosts, geographic distribution, seasonal occurrence and transmission of pathogens. Experimental and Applied Acarology, 27:113136.

HOWELL, C.J., WALKER, JANE B. \& NEVILL, E.M. 1978. Ticks, mites and insects infesting domestic animals in South Africa. Part 1. Descriptions and biology. Pretoria: Department of Agricultural Technical Services, Republic of South Africa (Science Bulletin, no. 393).

HOWELL, D.J., PETNEY, T.N. \& HORAK, I.G. 1989. The host status of the striped mouse, Rhabdomys pumilio, in relation to the tick vectors of heartwater in South Africa. Onderstepoort Journal of Veterinary Research, 56:289-291.

KEIRANS, J.E. 1993. Dermacentor rhinocerinus (Denny, 1843) (Acari: Ixodida: Ixodidae): redescription of the male, female and nymph and first description of the larva. Onderstepoort Journal of Veterinary Research, 60:59-68.

LEWIS, B.D., PENZHORN, B.L., LOPEZ-REBOLLAR, L.M. \& DE WAAL, D.T. 1996. Isolation of a South African vectorspecific strain of Babesia canis. Veterinary Parasitology, 63: 9-16.

MACIVOR, K.M. DE F. \& HORAK, I.G. 2003. Ixodid ticks of Angora and Boer goats, grysbok, common duikers, kudus and scrub hares in Valley Bushveld in the Eastern Cape Province. Onderstepoort Journal of Veterinary Research, 70:113-120.

NORVAL, R.A.I. 1984. The ticks of Zimbabwe. IX. Haemaphysalis leachi and Haemaphysalis spinulosa. Zimbabwe Veterinary Journal, 15:9-17.

PETNEY, T.N., HORAK, I.G., HOWELL, D.J. \& MEYER, S. 2004. Striped mice, Rhabdomys pumilio, and other murid rodents as hosts for immature ixodid ticks. Onderstepoort Journal of Veterinary Research, 71:313-318.

RECHAV, Y. 1982. Dynamics of tick populations (Acari: Ixodidae) in the eastern Cape Province of South Africa. Journal of Medical Entomology, 19:679-700.

RECHAV, Y., ZEEDERBERG, M.E. \& ZELLER, D.A. 1987. Dynamics of African tick (Acari: Ixodoidea) populations in a natural Crimean-Congo hemorrhagic fever focus. Journal of Medical Entomology, 24:575-583.

STAMPA, S. 1959. Tick paralysis in the Karoo areas of South Africa. Onderstepoort Journal of Veterinary Research, 28: 169-227 + 1 map.

WALKER, A.R., BOUATTOUR, A., CAMICAS, J.-L., ESTRADAPEÑA, A., HORAK, I.G., LATIF, A.A., PEGRAM, R.G. \& PRESTON, P. 2003. Ticks of domestic animals in Africa: a guide to identification of species. Edinburgh: Bioscience reports.

WALKER, JANE B., KEIRANS, J.E. \& HORAK, I.G. 2000. The genus Rhipicephalus (Acari: Ixodidae): a guide to the brown ticks of the world. Cambridge: Cambridge Academic Press. 